\title{
Laser-induced bubble dynamics inside and near a gap between a rigid boundary and an elastic membrane
}

\author{
Darja Horvat ${ }^{1}$, Uroš Orthaber ${ }^{2}$, Jörg Schille ${ }^{3}$, Lars Hartwig ${ }^{3}$, Udo Löschner ${ }^{3}$, \\ Andrej Vrečko ${ }^{2}$, Rok Petkovšek ${ }^{1}$
}

1 Faculty of Mechanical Engineering, University of Ljubljana, Aškerčeva 6, Ljubljana, Slovenia

2 Optotek d.o.o., Tehnološki park 21, Ljubljana, Slovenia

3 Faculty Mathematics / Sciences / Computer Science, Hochschule Mittweida - University of Applied

Sciences, Mittweida, Germany

\begin{abstract}
Aspects of laser-induced bubble dynamics in sample geometry closely resembling intraocular environment were studied. Laser pulses with a few millijoules of energy were focused into the sample, producing optical breakdown followed by formation of single bubbles. The sample consisted of a piece of polymethyl methacrylate (PMMA) material and a thin polyethylene (PE) elastic membrane which was carefully positioned parallel to the flat PMMA surface, creating a small and adjustable gap between them. The arrangement was placed in distilled water. Such geometry is frequently encountered in ophthalmology, specifically in posterior capsulotomy where there is a gap present between the intraocular lens and the posterior capsule. The formation and evolution of the laserinduced bubble was recorded with a high speed camera for different positions of the laser focus, i.e. inside the gap as well as outside the gap, in the water. The bubble dynamics was compared to the dynamics of the bubbles created near a rigid boundary alone and near an elastic membrane alone. The comparison showed that the evolution of the bubble created inside the gap can be described as a combination of the features which are characteristic for the rigid boundary and the elastic membrane cases, depending on relative dimensions of the gap and the bubble and on their relative positions. Possible influence on related medical procedures is discussed.
\end{abstract}

Keywords: bubble dynamics; laser induced bubble; boundary; membrane; gap; high speed photography

\section{Introduction}

Numerous laser medical procedures and other biomedical applications involve generation of bubbles in tissue, in various possible geometric configurations, e.g. bubbles near a rigid or elastic boundary or bubbles near a combined boundary, consisting of a rigid surface and an elastic membrane in close proximity (Walsh et al., 2011; Vogel and Venugopalan, 2011). Such complex boundary geometry occurs, for example, in posterior capsulotomy surgical procedure where a membrane-like elastic tissue called posterior capsule surrounds a rigid artificial intraocular lens. During the procedure the opacified elastic capsule is ruptured with a focused laser beam, improving the patient's vision (Aslam et al., 2003; Vogel et al., 1990). During the procedure a laser-induced bubble is formed inside or near a narrow gap between the intraocular lens and the posterior capsule. Understanding the 
mechanisms of laser-tissue interaction is of great importance for improving efficiency and safety of the procedure (Vogel et al., 1990). These mechanisms include dielectric breakdown and plasma formation in the focal area, subsequent bubble generation and growth, accompanied by shock wave emission and possible damage to the nearby tissue.

The bubble dynamics near a rigid boundary has been extensively studied by several authors (Vogel et al., 1989; Zhang et al., 1993; Blake and Gibson, 1987) as well as the bubble behavior near an elastic boundary (Brujan et al., 2001a and 2001b; Klaseboer et al., 2006; Ohl et al., 2009) and near an elastic membrane (Turangan et al., 2006; Orthaber et al., 2014; Aghdam et al., 2012). The case of bubble evolution near a membrane is in many aspects different from the one near an elastic boundary (Turangan et al., 2006). The thickness of the membrane is expected to influence the bubble dynamics and should therefore be considered.

No reports were found on study of bubble dynamics in the case of a complex double boundary, i.e. a thin membrane stretched parallel to a rigid boundary, as it is encountered in the human eye in the case of posterior capsulotomy. Single bubbles generated near a thin membrane were experimentally studied in (Orthaber et al., 2014) where it was recognized that additional research is needed to create experimental conditions as similar as possible to the real conditions inside the eye during the posterior capsulotomy procedure, mostly regarding the existence of a combined boundary, composed of solid material and elastic membrane.

After expansion to its maximum diameter, the laser-induced bubble collapses, then expands again and often goes through more than one such cycle. When the bubble is situated near a boundary, liquid jets are generally created during the bubble collapse which are directed towards the boundary in the case of a rigid boundary and away from it in the case of a free surface (Turangan et al., 2006). The dynamics depends on parameters such as maximum bubble diameter and distance between the bubble and the boundary as well as on the material properties. In an extended study of bubble interaction with elastic boundary (Brujan et al., 2001a and 2001b) complex behavior was described, such as boundary deformation, bubble evolution into mushroom shaped formation and bubble splitting. Similar behavior was observed near a relatively thick membranes (Turangan et al., 2006; Aghdam et al., 2012) and near a very thin membrane (a few micrometers) in (Orthaber et al., 2014). The latter study showed how the cavitation bubble expansion and collapse causes significant deformation of the thin membrane and even its rupture. The behavior of the bubble created at different distances from the membrane was analyzed, revealing that the process is most destructive to the membrane at intermediate distances when the distance between the membrane and the bubble center was smaller than $70 \%$ of the bubble maximum radius. In this case the rupture produced in the membrane is largest and has well defined borders.

The present paper addresses the question of bubble dynamics near a complex boundary, which has characteristics both of the elastic membrane and of the rigid boundary. The goal of the study is to assess which of the two boundaries has more influence on the bubble dynamics, how the bubble dynamics is changed in the presence of the gap between them and how it is influenced by the gap width.

\section{Experimental system}

To gain further insight into the real processes in the eye during the capsulotomy procedure, a polyethylene (PE) elastic membrane was placed in close proximity (a few hundreds of microns) of a rigid boundary made of polymethyl methacrylate (PMMA) material, representing the intraocular lens. We experimentally analyzed the behavior of the bubble in the vicinity of this complex boundary, for different relative positions of the bubble with respect to the boundary. The analysis of the bubble dynamics in the vicinity of such a combined boundary is intended to contribute to accurate description of the medical procedure. 
The experimental setup is shown schematically in Fig. 1. A Nd:YAG laser was used with pulse duration of approximately $5 \mathrm{~ns}$ (FWHM), at $2.5 \mathrm{~mJ}$ and $10 \mathrm{~mJ}$ of energy. The beam was first expanded to a diameter of approximately $40 \mathrm{~mm}$ and then tightly focused with a lens of $115 \mathrm{~mm}$ focal length to a diameter of a few micrometers into the sample. Optical breakdown occurred in the focal area, followed by bubble formation. The pulse energy was adjusted with a custom-built attenuator and measured with a calibrated energy meter.

The evolution of the laser-induced cavitation bubble inside the sample was recorded with a highspeed camera (HSFC PRO, 12 bit ultra-speed intensified imaging) with a frame rate of 100 kiloframes/s. Pulses from a $100 \mathrm{~W}$ laser diode with a wavelength of $808 \mathrm{~nm}$ were synchronized with the high-speed camera to serve as illumination. The time between consecutive frames was $10 \mu$ s and in some cases $5 \mu \mathrm{s}$.

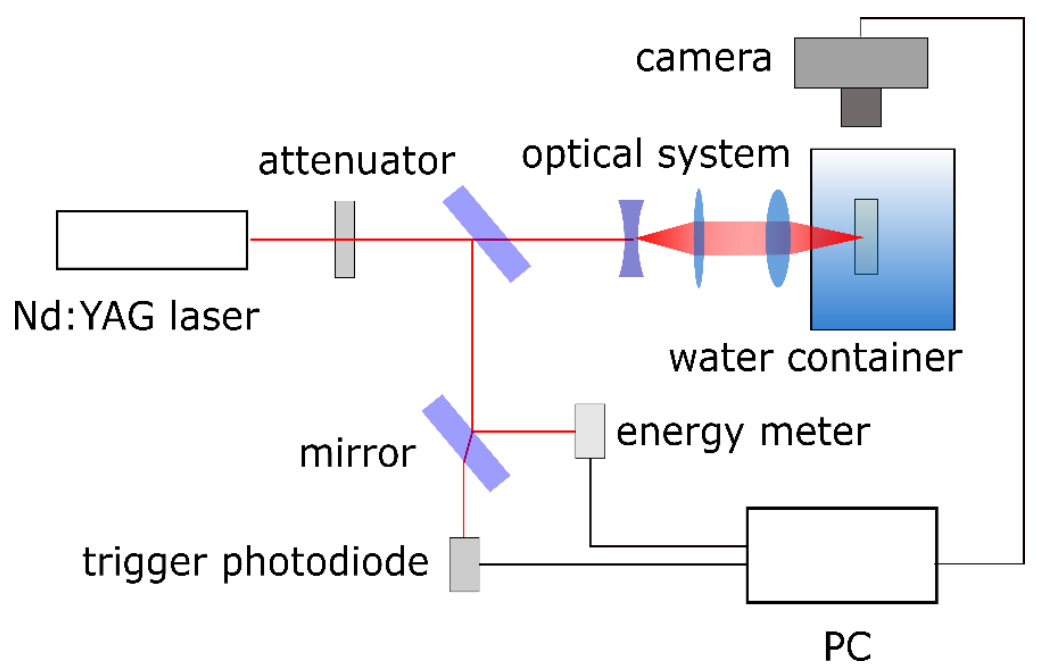

Figure 1 Experimental system

\section{a)}

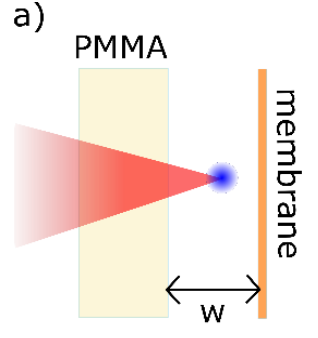

b)

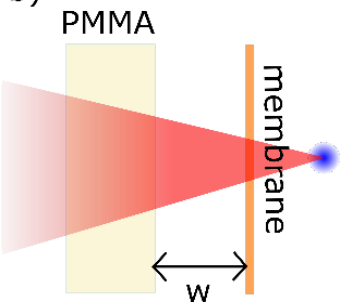

Figure 2 Sample geometry. The laser beam is coming from the left and is focused a) inside the gap of width $w$ between the PMMA and the membrane and $b$ ) to the right of the membrane, outside the gap.

The key component of the experimental system was the sample inside the water-filled glass container, designed with the intention to mimic the real conditions inside the human eye during the 
posterior capsulotomy. It consisted of a PMMA block representing the intraocular lens and an approximately $6 \mu \mathrm{m}$ thick PE membrane, representing the posterior capsule. The membrane was placed parallel to the PMMA boundary, leaving a small gap between the PMMA and the PE membrane. The tension in the membrane, which was uniformly stretched in the radial direction, was approximately $2 \mathrm{~N} / \mathrm{m}$. The Young's modulus of the PE membrane was $0.24 \mathrm{GPa}$. The sample was placed in the center of the container, approximately $3 \mathrm{~cm}$ below the water surface. The gap between the PMMA and the PE membrane was adjustable and was set at 300, 800 and $1600 \mu \mathrm{m}$. The laser beam was precisely focused at various positions outside and inside the gap. The described sample geometry is shown in detail in Fig. 2.

\section{Results and discussion}

Several sequences were recorded with the described experimental setup, with different parameters. Results are presented here for laser pulse energies of 2.5 and $10 \mathrm{~mJ}$, gap width of 300, 800 and $1600 \mu \mathrm{m}$ and several relative positions of the bubble with respect to the boundary.

Sequences showing the dynamics of laser-induced bubbles and membrane deformation and rupture were analyzed and compared to previous findings. To describe the relative position and size of the bubble with respect to the boundary a parameter $\gamma$ is defined:

$$
\gamma=\frac{L}{R_{\max }}
$$

where $L$ is the distance between the bubble center and the boundary and $R_{\max }$ is the maximum bubble radius (Fig. 3a).

The first sequences were recorded in the presence of the rigid boundary only, without the membrane. The dynamics of the bubble near a rigid boundary is relatively well known from previous work (Blake et al., 1987; Zhang et al., 1993). The collapse of the bubble is accompanied by a jet, directed towards the boundary. Such dynamics was also recorded in our measurements, confirming the basic features of the dynamics of the rigid boundary case (Fig. 4 and Fig. 5.), although the jet can be clearly seen only in the sequence in Fig. 4, where the $\gamma$ coefficient is large. The graphics, superimposed on the first frame shown in Fig. 4 depicts the geometry of the experimental arrangement: the laser beam was focused through the transparent PMMA material from the left. The same geometry was used throughout the photographic material presented in this article.

The dynamics of the bubble formed near a membrane has been studied numerically and experimentally (Turangan et al., 2006; Aghdam et al., 2012; Shervani-Tabar et al., 2013) for spark generated bubbles and by (Orthaber et al., 2014) for laser-induced bubbles. In the work by Orthaber et al., roughly three regimes were identified with respect to the bubble-membrane distance. At large distances $(\gamma>>1)$, there was little interaction between the bubble and the membrane and effects like membrane deformation and jet formation were not very pronounced. On the other hand, when the laser focus was at the membrane $(\gamma \approx 0)$, the laser induced plasma ruptured the membrane even before the formation of the bubble. Like in the case of $y \gg>1$, no substantial pull on the membrane was observed. Most complex was the dynamics at intermediate distances $(\gamma \approx 1)$. After the creation of the bubble the membrane was pushed away by the growing bubble. This was followed by a strong pull of the membrane in the bubble collapse phase. During the collapse the bubble transformed from mushroom-like shape into hourglass formation and finally split into two smaller bubbles. A jet was formed, directed towards the membrane and the distant of the two created bubbles oscillated a few more times while migrating away from the membrane. It was established that in this regime the circumstances were the most appropriate to cause largest rupture of membrane, a consequence of 
the strong pull during the bubble collapse. The observation in (Orthaber et al., 2014) is in accordance with the calculations in (Turangan et al., 2006) and (Shervani-Tabar et al., 2013).

In our experiments the bubbles were also created near a membrane but with the addition of a rigid boundary in close proximity. The laser-induced bubbles were formed inside (Fig. 2a) as well as outside the gap (Fig. 2b).

a)

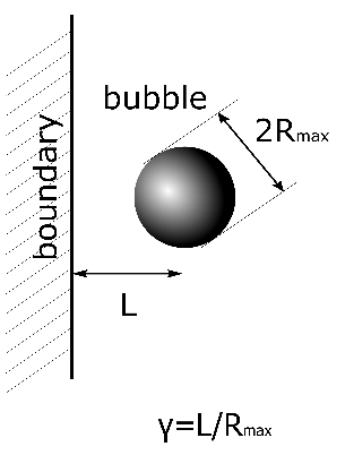

b)

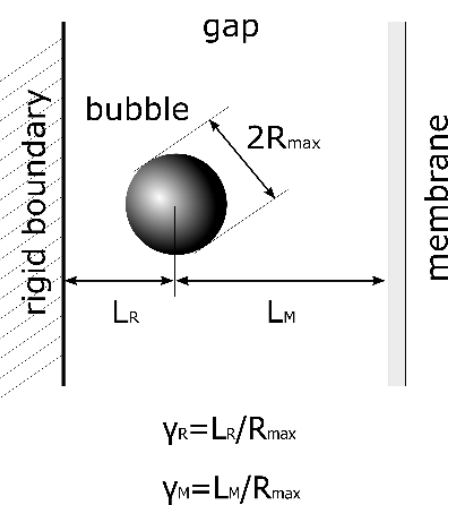

Figure 3 The definition of a) the parameter $\gamma$ for the bubble in the vicinity of a simple boundary and $b$ ) the parameters $\gamma_{M}$ and $\gamma_{R}$ for the bubble in the vicinity of a double boundary
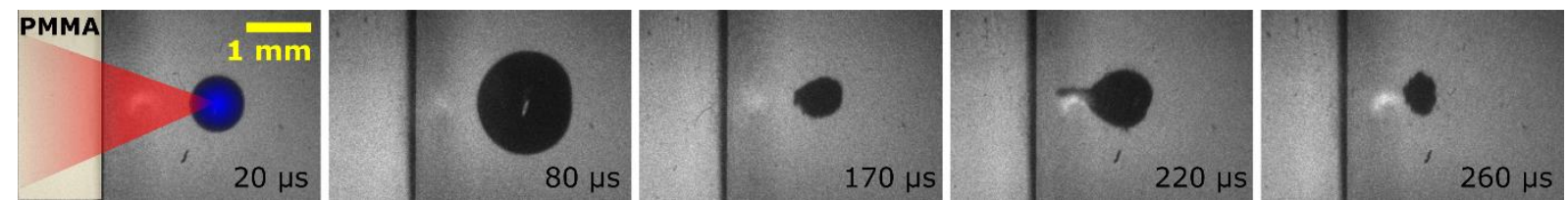

Figure 4 Bubble dynamics (selected frames) far from rigid boundary (PMMA), pulse energy 2.5 $\mathrm{mJ}, \gamma=2.3$. The geometry of the experimental arrangement is superimposed in the first frame, depicting the position of the PMMA material and showing the path of the incoming laser beam, from left.
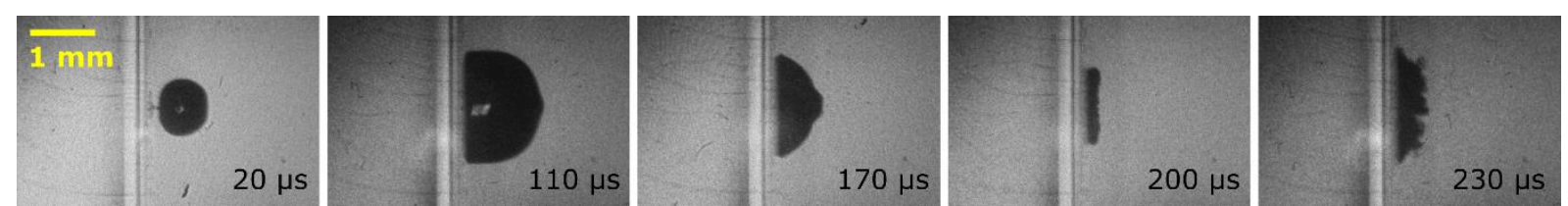

Figure 5 Bubble dynamics (selected frames) near rigid boundary, $\gamma=0.6$. Same geometry as in Fig. 4.

\subsection{Bubble formed outside the gap}

First we consider the case where the bubble maximum diameter and the width of the gap are of comparable size.

Figs. 6 and 7 show dynamics of bubbles created with a $2.5 \mathrm{~mJ}$ laser pulse outside the gap, $1400 \mu \mathrm{m}$ and $500 \mu \mathrm{m}$ away from the membrane, respectively. The gap width was $800 \mu \mathrm{m}$ and the maximum bubble diameter $1500 \mu \mathrm{m}$, giving values $\gamma=2.0$ (Fig. 6) and $\gamma=0.7$ (Fig. 7). 
The bubble in Fig. 6 was positioned so far away from the combined boundary that the boundary had only little influence on bubble dynamics. The presence of the boundary introduced some asymmetry to the bubble shape in the collapse phase but the influence was very weak and no jet was observed. The evolution was similar to the sequence in Fig. 4 where the bubble was created far away from the rigid boundary, with no membrane present. The case in Fig. 7 was more complex and can be directly compared to a sequence in (Orthaber et al., 2014) occurring at a membrane alone, having the same coefficient $(\gamma=0.68)$ and described as "best case scenario" regarding the creation of a rupture in the membrane. As in (Orthaber et al., 2014), the bubble in the expansion phase pushed away the membrane and was flattened on the side where it touched the membrane. During the collapse phase a mushroom shape was formed, similar to the corresponding case in (Orthaber et al., 2014). At that point obviously a rupture in the membrane occurred (Fig. 7, frame 4) since the bubble was transferred completely inside the gap and continued its evolution there. At this point the resemblance to the case of single membrane in (Orthaber et al., 2014) ends. We suggest that because of the geometry the negative pressure difference that was created in the collapse phase on the other side of the membrane (with respect to the bubble position) i.e. inside the gap, was locally substantially larger than in the case of single membrane. This resulted in pulling and rupture of the membrane, followed by dragging the bubble through the rupture. Once inside the gap, the bubble experienced a few more cycles, gradually dissipated its energy and disintegrated during the process.
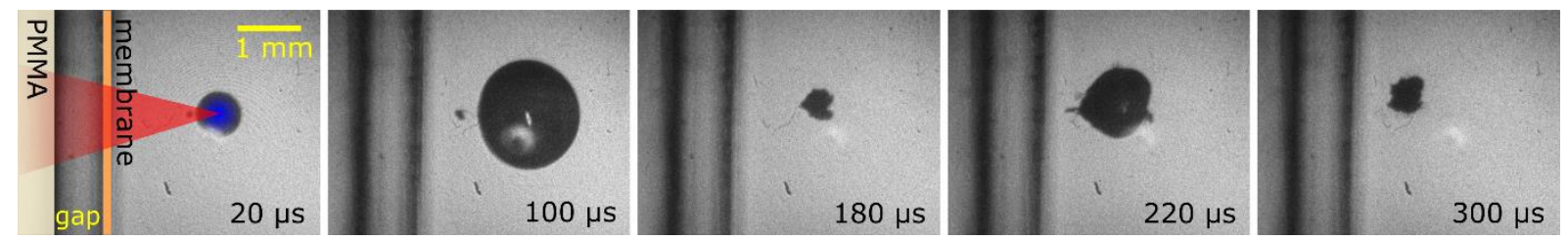

Figure 6 Bubble dynamics outside the gap (selected frames), pulse energy $2.5 \mathrm{~mJ}$, gap width $800 \mu \mathrm{m}, \gamma=2.0$. The geometry of the experimental arrangement is superimposed in the first frame, depicting the position of the PMMA material and the PE membrane together with the path of the incoming laser beam, from the left.
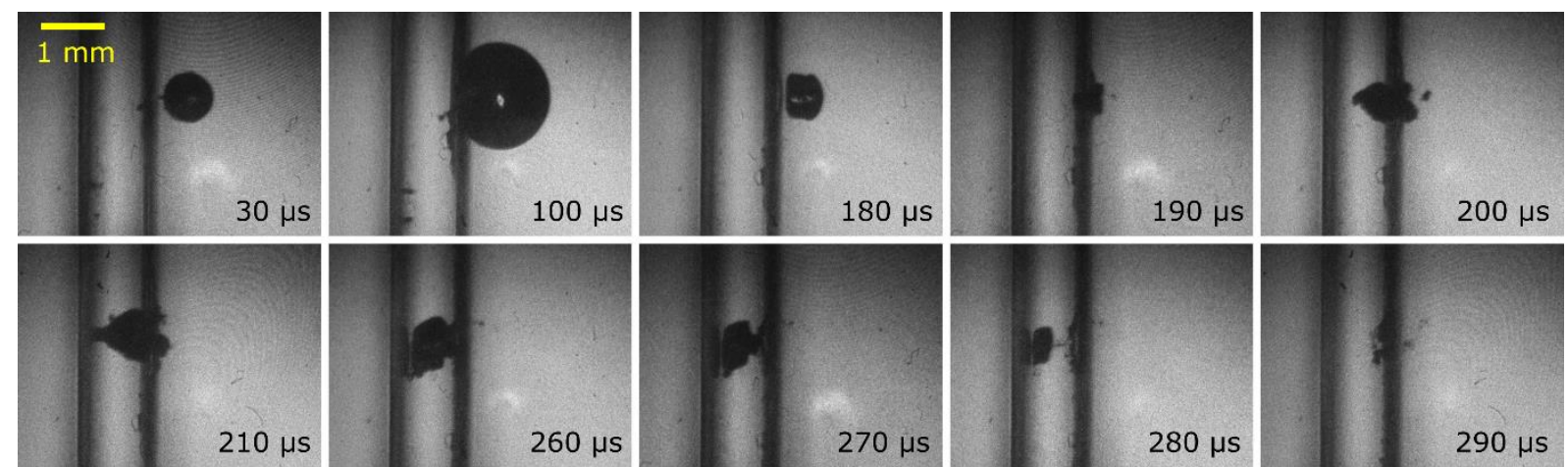

Figure 7 Bubble dynamics outside the gap (selected frames), pulse energy $2.5 \mathrm{~mJ}$, gap width $800 \mu \mathrm{m}, \mathrm{\nu}=0.7$. Same geometry as in Fig. 6 .

\subsection{Bubble formed inside the gap}

Figs. 8 and 9 show dynamics of a bubble created inside the $1600 \mu \mathrm{m}$ gap. In the case of a bubble inside the gap we define two parameters, $\gamma_{M}$ and $\gamma_{R}$, describing the relative bubble position with respect to the membrane and to the rigid boundary, respectively (Fig. 3b):

$$
\gamma_{M}=\frac{L_{M}}{R_{\max }} \text { and } \gamma_{R}=\frac{L_{R}}{R_{\max }} .
$$


As before, $R_{\max }$ is the maximum bubble radius, while $L_{M}$ and $L_{R}$ are the distances of the bubble center from the membrane and from the rigid boundary, respectively.

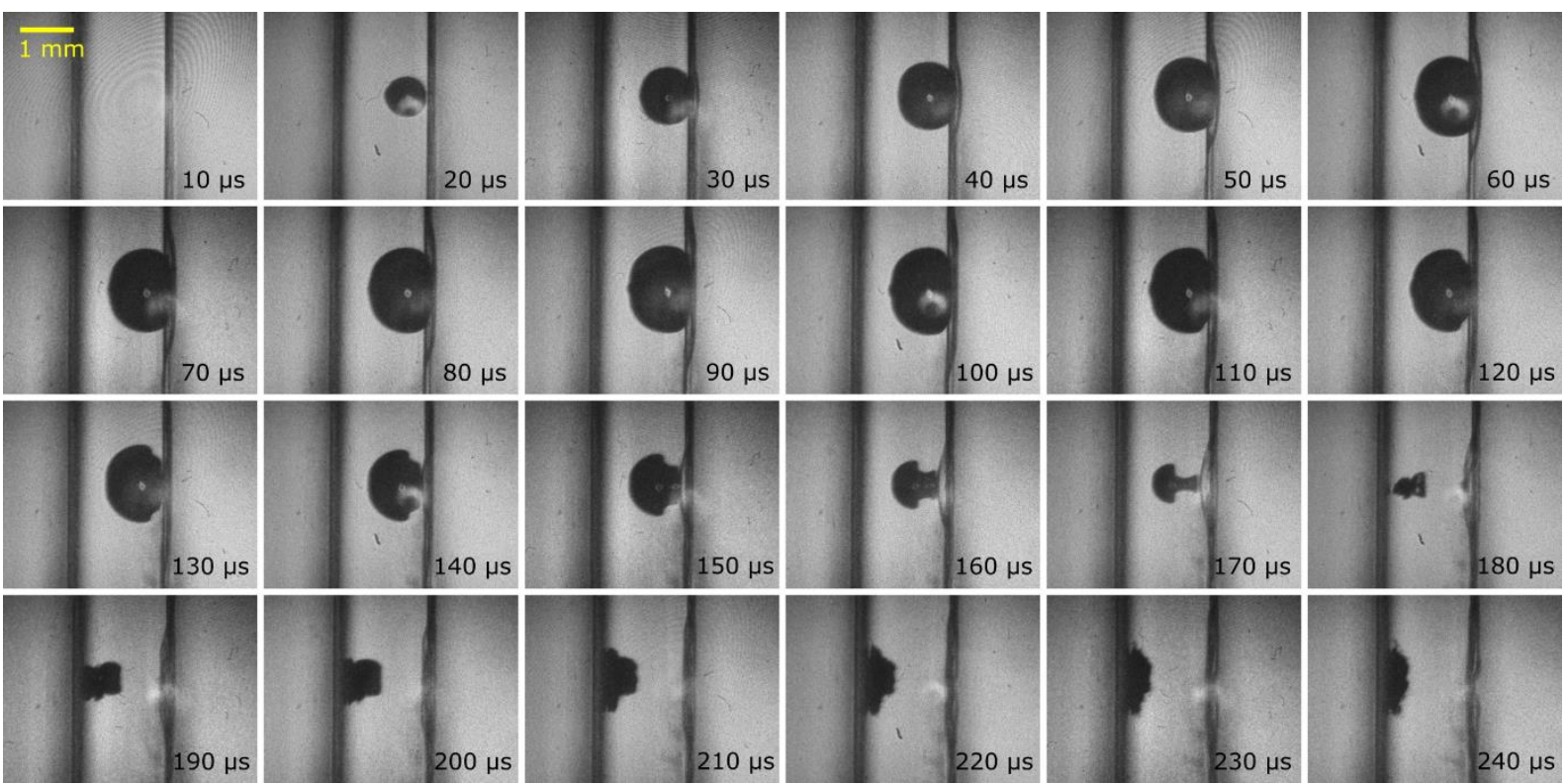

Figure 8 Bubble dynamics inside the gap, near the membrane, pulse energy $2.5 \mathrm{~mJ}$, gap width $1600 \mu \mathrm{m}, \gamma_{\mathrm{R}}=1.5, \gamma_{\mathrm{M}}=0.5$.

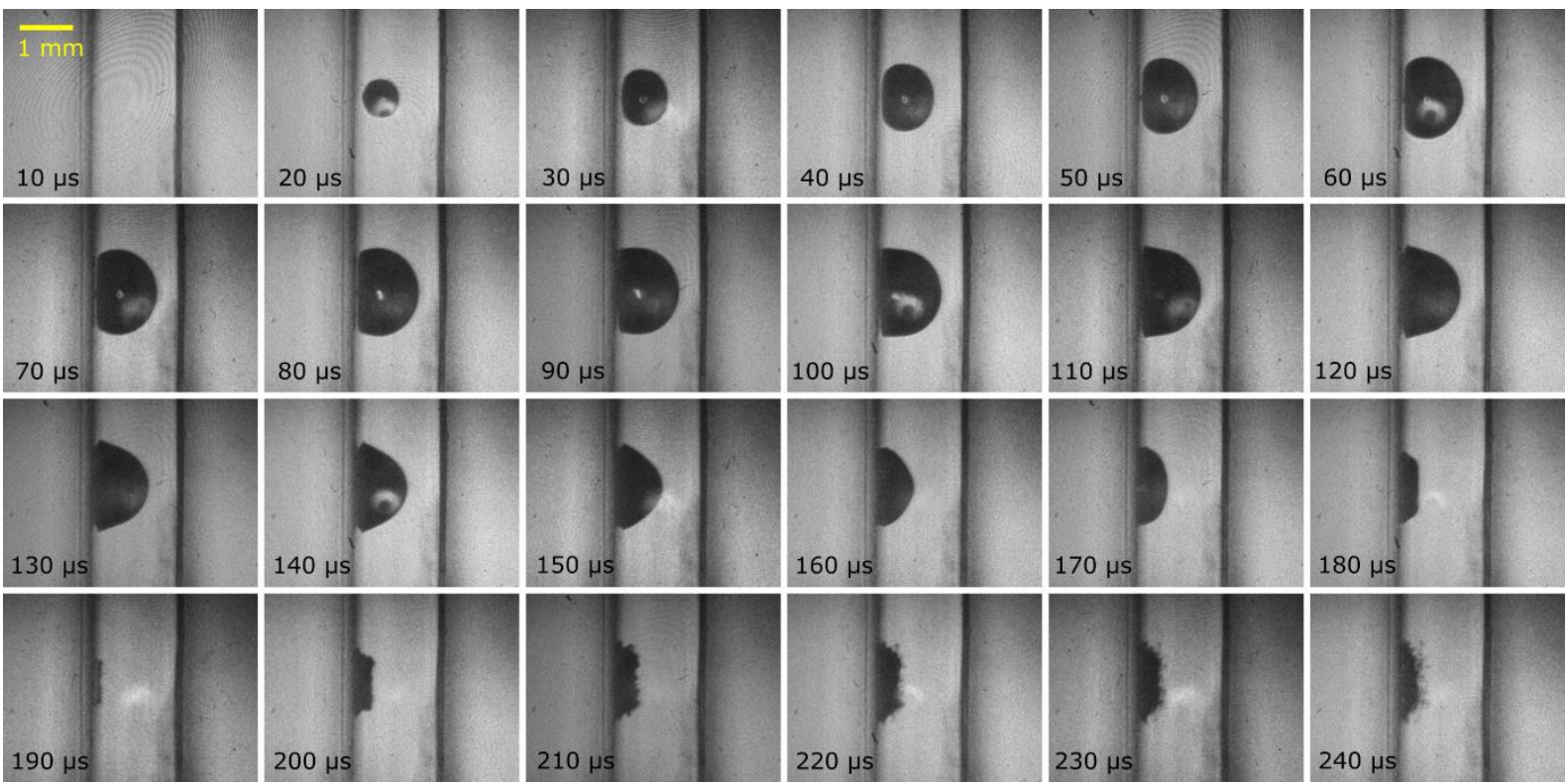

Figure 9 Bubble dynamics inside the gap, near PMMA, pulse energy $2.5 \mathrm{~mJ}$, gap width $1600 \mu \mathrm{m}$, $\gamma_{R}=0.6, \gamma_{M}=1.3$. 
In the sequence in Fig. 8 the bubble center was situated closer the membrane, with $\gamma_{R}=1.5$ and $\gamma_{M}=0.5$, while in the sequence in Fig. 9 the bubble center was located closer to the rigid boundary with $\gamma_{R}=0.6$ and $\gamma_{M}=1.3$. Although in both cases the bubble center was close to the center of the gap, the resulting bubble dynamics was quite different for the two cases, especially after the maximum bubble size was reached. The beginning of sequence in Fig. 8 (upper row) shows membrane deformation away from the bubble during the bubble growth and subsequent mushroom shape formation (third row), followed by strong deformation of the membrane and possible rupture during the bubble collapse. This is similar to the dynamics of a bubble near the membrane alone (Orthaber et al., 2014) with the typical mushroom shape in the collapse phase. Unlike the case in Fig. 7, the bubble did not penetrate the membrane. Rather, it stayed on the same side of the membrane. Eventually, as the bubble migrated away from the membrane, it collided with the rigid wall.

On the other hand, in the sequence in Fig. 9 where the center of the bubble was positioned only a little closer to the rigid boundary than in the case of Fig. 8, it exhibited a notably different evolution, resembling more the sequence in Fig. 5, with similar parameters but without the membrane present. The bubble did not reach the membrane in the growth phase and its dynamics resembled the dynamics of a bubble near a rigid wall. It grew in a hemispherical shape, then collapsed and disintegrated at the rigid boundary. In this case the membrane did not seem to have a substantial influence on the bubble shape.

When a relatively small bubble was created inside the gap the dynamics of the bubble was greatly influenced by its position relative to the rigid boundary or the membrane. For the parameters in this experiment the region of influence of each of the two boundaries extended approximately to the center of the gap between them.

\subsection{Bubble large compared to the width of the gap}

Figs. 10 and 11 show the sequences in the case of a bubble which was large compared to the width of the gap. In this case the bubbles were created with a $10 \mathrm{~mJ}$ laser pulse, so their maximum radius was larger and the dynamics was slower. The oscillation time of the bubble is namely proportional to the maximum radius of the bubble (Brujan et al., 2001b), so characteristic growth time is longer for a larger bubble.

In Fig. 10 we observe a sequence with the large bubble situated outside the gap, with $\gamma=0.9$. The dynamics was similar to the dynamics of a bubble near a rigid boundary, with only negligible influence of the membrane and the gap which was in this case small compared to the bubble.

Various features can be observed in the case where the center of the large bubble was positioned in the middle of the gap, with $\gamma_{M}=\gamma_{R}=0.63$, as in Fig. 11. During the growth the portions of the bubble were shaped as if there was a plane dividing it along the gap parallel to the boundaries, with the left half (close to the rigid boundary) obeying the corresponding growth dynamics at the rigid boundary and the right half (close to the membrane) pushing away the membrane, as in the case of bubble dynamics near the membrane alone. Upon reaching the maximum size the bubble began to collapse, still following the dual behavior: the left half collapsed as in the case of a bubble very near a rigid wall, disintegrating in the final phase into irregularly shaped collapse products, while the right half, near the membrane, strongly pulled the membrane inwards and formed the corresponding bottom part of the mushroom formation as in the case of a bubble close to a membrane alone. Through the whole process the plane along the center of the gap remained dividing the bubble into two parts, as a consequence of the influence of the two different boundaries. In continuation, as the disintegration products remained visible at the rigid boundary, a relatively strong wavelike motion of the membrane can be observed after the part of the bubble at the membrane had collapsed. As in the case of the smaller bubbles we describe the situation as the region of influence for each of the two boundaries, extending approximately to the middle of the gap. 
Fig. 12 shows part of the sequence of the bubble dynamics inside the gap around the moment of bubble splitting, close to $300 \mu \mathrm{s}$ from the bubble generation. Framing rate here was increased to $200 \mathrm{kframes} / \mathrm{s}$ so that the time between consecutive frames was $5 \mu \mathrm{s}$. It can be noticed that during the bubble collapse a waist was formed on the bubble, which was a result of the radial flow of the liquid towards the center of the bubble along the curved surface of the deformed membrane. This flow eventually caused the bubble to split (last frame in Figure 12).

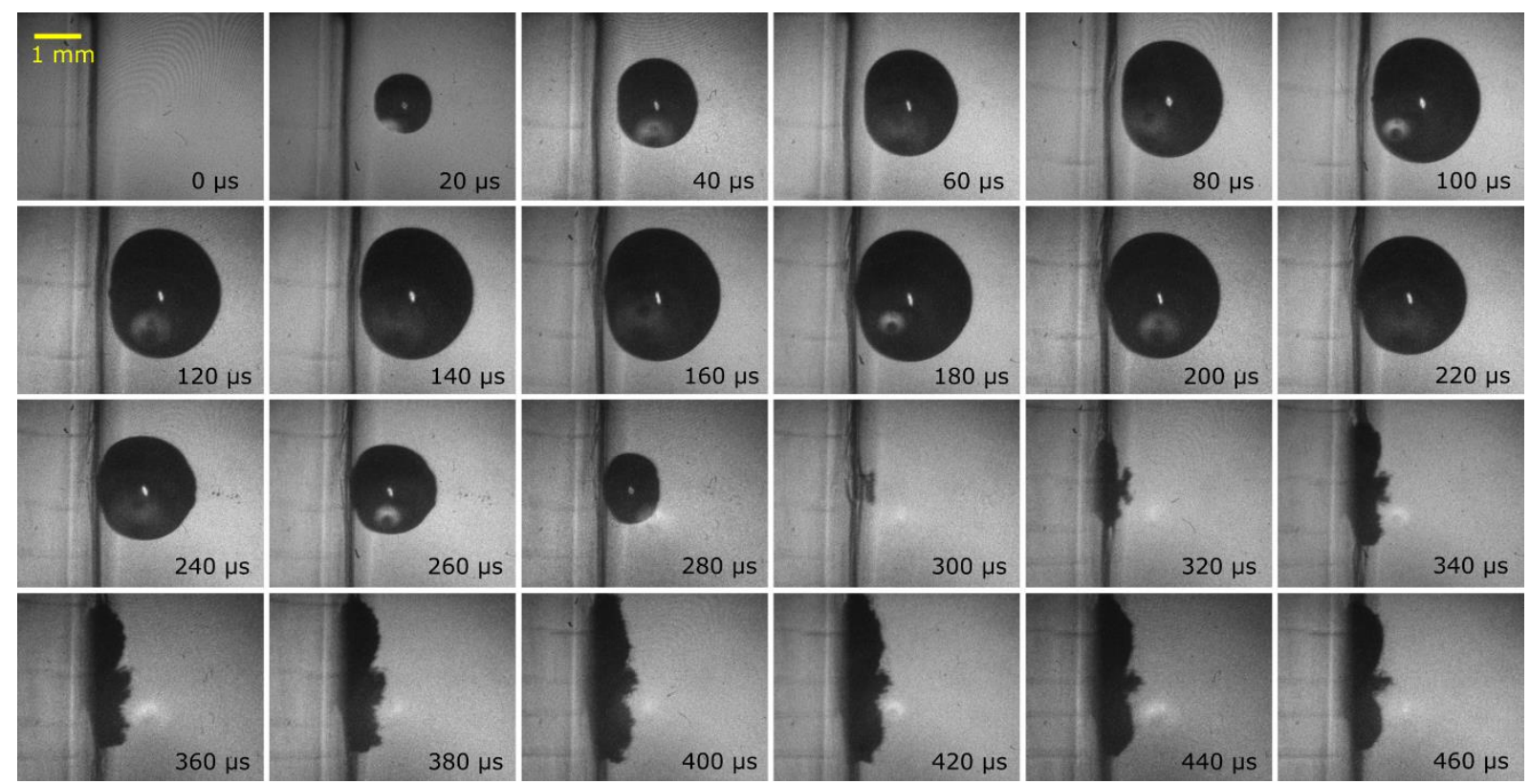

Figure 10 Bubble dynamics for a bubble large compared to the width of the gap, positioned outside the gap, pulse energy $10 \mathrm{~mJ}$, gap width $300 \mu \mathrm{m}, \gamma_{\mathrm{M}}=0.9$.
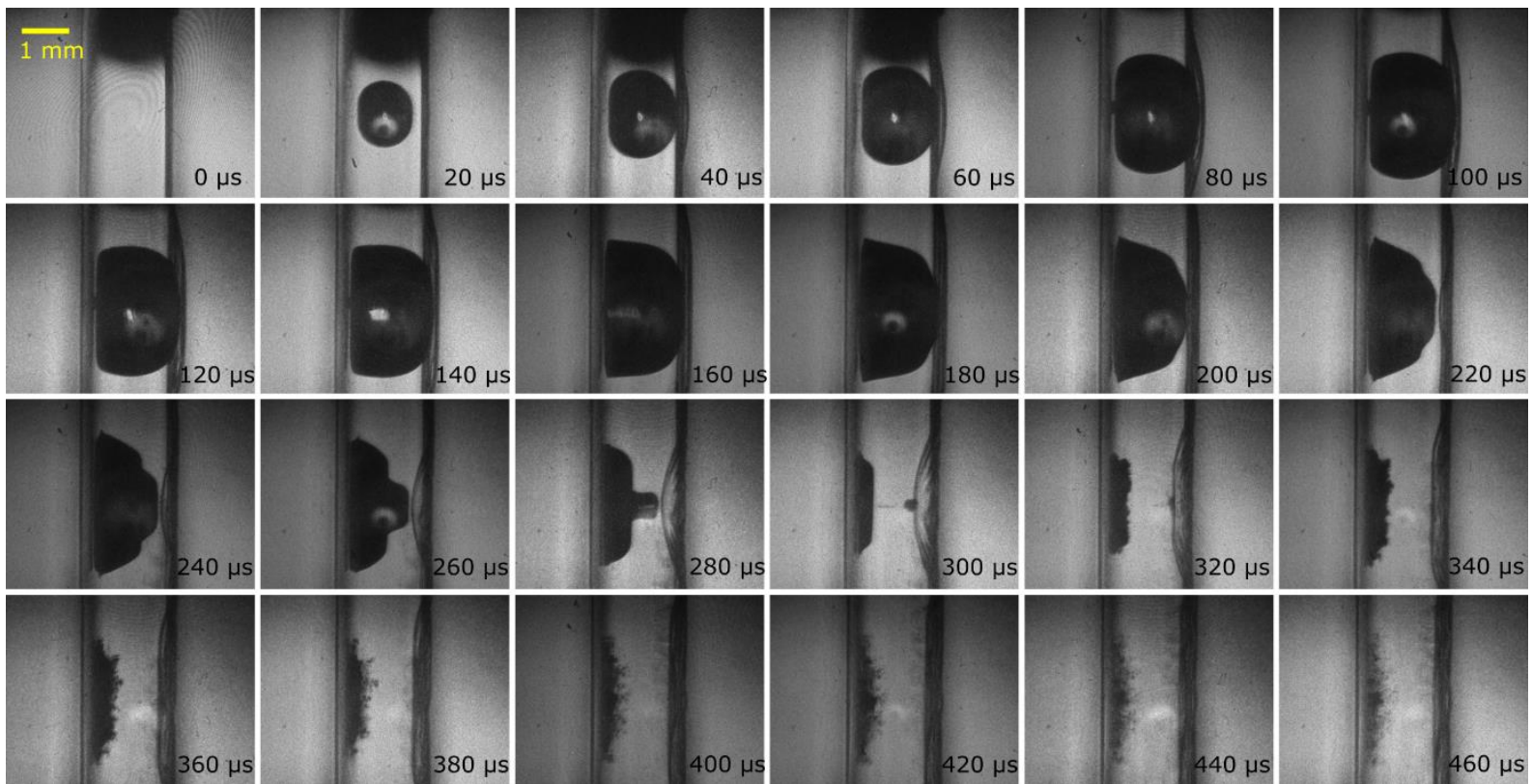

Figure 11 Bubble dynamics for a bubble large compared to the width of the gap, positioned inside the gap, pulse energy $10 \mathrm{~mJ}$, gap width $1600 \mu \mathrm{m}, \gamma_{M}=\gamma_{R}=0.63$. 

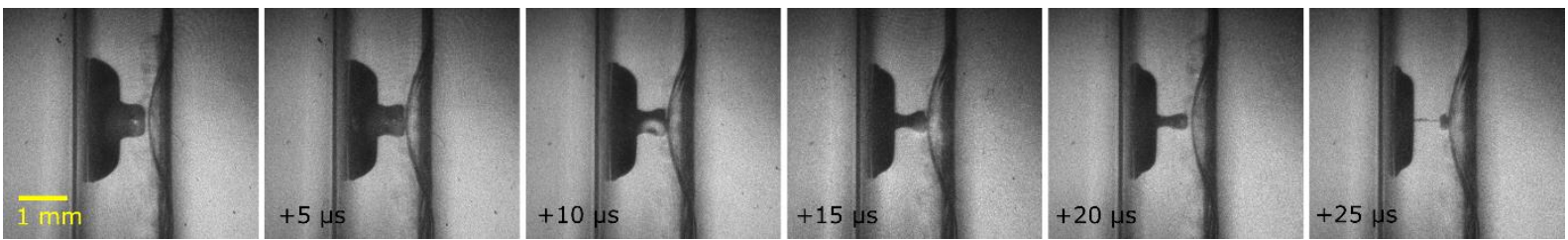

Figure 12 Part of the sequence with the same parameters as in Fig. 11 (bubble inside a gap of $1600 \mu \mathrm{m}$ width, pulse energy $10 \mathrm{~mJ}, \gamma_{M}=\gamma_{R}=0.63$ ), around the time of bubble splitting. The time between consecutive frames is $5 \mu \mathrm{s}$.

Table 1

\begin{tabular}{|c|c|c|c|c|c|c|}
\hline $\begin{array}{c}\text { Fig. } \\
\text { No. }\end{array}$ & $\begin{array}{c}\text { Gap width } \\
(\mathrm{mm})\end{array}$ & $\begin{array}{c}\text { Laser pulse } \\
\text { energy (mJ) }\end{array}$ & $\begin{array}{c}\text { Bubble size } \\
\text { (parallel } \mathrm{x} \\
\text { perpendicular) }\end{array}$ & $\gamma / \gamma_{\mathrm{R}} / \mathrm{\gamma}_{\mathrm{M}}$ & $\begin{array}{c}\text { Membrane } \\
\text { deformation } \\
\text { at bubble } \\
\text { growth } \\
(\mathrm{mm})\end{array}$ & $\begin{array}{c}\text { Membrane } \\
\text { deformation } \\
\text { at bubble } \\
\text { collapse } \\
(\mathrm{mm})\end{array}$ \\
\hline 4 & - & 2.5 & $1.45 \times 1.60$ & $2.3 /-/-$ & & \\
\hline 5 & - & 2.5 & $1.25 \times 1.75$ & $0.6 /-/-$ & & \\
\hline 6 & 0.80 & 2.5 & $1.55 \times 1.55$ & $2.0 /-/-$ & & \\
\hline 7 & 0.80 & 2.5 & $1.40 \times 1.65$ & $0.7 /-/-$ & & 0.30 \\
\hline 8 & 1.60 & 2.5 & $1.20 \times 1.60$ & $-/ 1.5 / 0.5$ & 0.20 & \\
\hline 9 & 1.60 & 2.5 & $1.20 \times 1.60$ & $-/ 0.6 / 1.3$ & & 0.20 \\
\hline 10 & 0.30 & 10 & $2.4 \times 2.8$ & $-/-/ 0.9$ & 0.20 & 0.45 \\
\hline 11 & 1.60 & 10 & $1.8 \times 2.9$ & $-/ 0.63 / 0.63$ & 0.30 & \\
\hline
\end{tabular}

Various data collected from Figs. 4 through 11 is organized in Table 1: gap width, laser pulse energy, bubble size, coefficient gamma regarding bubble position as well as the maximum membrane displacement in the bubble growth and bubble collapse phases.

\subsection{Discussion}

From the presented cases of bubble dynamics it can be concluded that the evolution of a bubble near the combined boundary having the described geometry is influenced by the relative bubble-gap size and the relative bubble-gap position. These two parameters influence the shape of the dynamic pressure field generated inside the gap and in its vicinity during the bubble growth and collapse. This nonhomogeneous pressure field governs locally the behavior of the different parts of the bubble. In the presented experimental conditions this results in the bubble behavior as if effectively each of the two boundaries predominantly influenced the pressure field on its half of the gap.

There are noticeable differences between bubble dynamics and its effect on the membrane in cases when the bubble is located inside or outside the gap. Considering the case when the bubble is situated outside the gap, the dynamics resembles the bubble dynamics near a rigid boundary if the gap is narrow compared to the maximum bubble radius. If, on the other hand, the gap is large compared to the bubble size, the bubble outside of the gap evolves as if it was situated next to the membrane alone.

When a bubble is generated inside the gap, but closer to the membrane, the evolution resembles the dynamics exhibited near the membrane alone with the difference that the jet strikes in 
the "wrong" direction against the rigid boundary, rather than towards the membrane. However, if the bubble inside the gap is situated closer to the rigid boundary, the influence of the presence of the membrane is very weak.

The recorded sequences suggest that the influence of the membrane on the bubble dynamics is significant when the corresponding ratios $\gamma$ (in the case of bubble located outside the gap) or $\gamma_{M}$ (in the case of bubble located inside the gap) are smaller than 1 . The influence drops rapidly with increasing $\gamma$ or $\gamma_{\mathrm{M}}$. Furthermore, comparison of Figs. 8 and 9 shows that a quite small difference in the position of the bubble center when the bubble is inside the gap results in completely different bubble dynamics. Also, for the bubble, which is large compared to the gap size in Fig. 11 a remarkable feature can be observed where each of the two boundaries seem to determine the evolution for their corresponding half of the bubble and the transition between the two regions of influence is very sharp. As suggested above, the local behavior of the bubble is a consequence of the complex dynamic pressure generated in the presence of the combined boundary by the bubble itself.

The outcome of the medical procedure depends on various parameters such as the laser pulse energy i.e. bubble size, gap width, laser focus position and their interplay. The knowledge of optimal parameters for the surgical procedure i.e. rupture of the posterior capsule enables the operator to use the smallest possible laser pulse energy. This is advantageous because it results in substantially smaller plasma of the laser-induced breakdown, thus reducing the risk of the unwanted damage of the intraocular lens by the plasma often referred to as lens-pitting.

In the study of laser capsulotomy by (Hawlina et al., 2016) the authors showed that in the case of a wider gap between the intraocular lens and the posterior capsule fewer laser pulses were needed to achieve the desired rupture of the capsule. This observation is consistent with the findings of this paper, where most pronounced interaction between the bubble and the membrane was observed in the case gap size which was large compared to bubble radius. This was true in both cases, when the bubble was located inside and when it was located outside the gap, as long as it was close to the membrane $\left(\gamma<1\right.$ or $\left.\gamma_{M}<1\right)$. However, in the case of the bubble inside the gap the interaction appears somewhat stronger (Fig. 7 versus Fig. 8). It seems that the presence of the rigid boundary and the liquid inside the gap inhibits the movement of the membrane compared to the case of a membrane alone. When the bubble is located inside the gap there is no rigid barrier on the other side of the membrane, thus allowing a stronger membrane movement. Assuming that a more dynamic movement with excessive membrane amplitudes can cause the rupture of the membrane it is implied that the same bubble in the vicinity of a wider gap, especially if located inside the gap, is more likely to damage the membrane than if the gap is narrow.

\section{Conclusion}

Bubble dynamics was studied experimentally for the specific case of laser-induced bubbles created in the sample geometry that closely resembles the conditions in posterior capsulotomy surgical procedure. The aim was to record for the first time the bubble evolution in realistic conditions in the vicinity of the membrane near a rigid boundary. Several cases were qualitatively examined. It was confirmed that the bubble evolution is highly dependent on bubble position with respect to both boundaries, the membrane and the rigid boundary. The inside of the gap could be described as having two regions of influence, for the parameters used in the study each region extending to approximately half the width gap, where the influence of the other, distant boundary is very small. This observation holds for both the bubbles smaller than the width of the gap and for bubbles approximately twice the width of the gap.

The new insight into both the bubble and the membrane dynamics is consistent with studies of laser capsulotomy regarding the effect of the gap width on the procedure. Further developed, this new 
knowledge will contribute to better performance and outcome of medical procedures and prevention of unwanted damage to intraocular lens, thus improving the overall performance and reliability.

\section{Acknowledgments}

This research was supported by the Slovenian Research Agency (project no. L2-6780 and program no. P2-0392).

\section{References}

Aghdam H.A., Ohl S.W., Khoo B.C., Shervani-Tabar M.T., Nobari M.R.H., 2012. Effect of the viscosity on the behavior of a single bubble near a membrane. International Journal of Multiphase Flow 47, 17-24.

Aslam T.M., Devlin H., Dhillon B., 2003. Use of Nd:YAG laser capsulotomy. Survey of Ophthalmology 48(6), 594-612.

Blake J.R., Gibson D.C., 1987. Cavitation bubbles near boundaries. Ann. Rev. Fluid. Mech. 19, 99123.

Brujan, E.A., Nahen, K., Schmidt, P., Vogel, A., 2001a. Dynamics of laser-induced cavitation bubbles near an elastic boundary. J. Fluid Mech. 433, 251-281.

Brujan, E.A., Nahen, K., Schmidt, P., Vogel, A., 2001b. Dynamics of laser-induced cavitation bubbles near elastic boundaries: influence of the elastic modulus. J. Fluid Mech. 433, 283-314.

Hawlina G., Drnovšek-Olup B., Možina J., Gregorčič P., 2016. Photodisruption of a thin membrane near a solid boundary: an in vitro study of laser capsulotomy. Appl. Phys. A 122:118. DOI 10.1007/s00339-016-9648-z.

Klaseboer E., Turangan C.K., Khoo B.C., 2006. Dynamic behaviour of a bubble near an elastic infinite interface. International Journal of Multiphase Flow 32, 1110-1122.

Ohl S.W., Klaseboer E., Khoo B.C., 2009. The dynamics of non-equilibrium bubble near biomaterials. Phys. Med. Biol. 54, 6313-6336.

Orthaber U, Petkovšek R., Schille J., Hartwig L., Hawlina G., Drnovšek-Olup B., Vrečko A., Poberaj I., 2014. Effect of laser-induced cavitation bubble on a thin elastic membrane. Optics \& Laser Technology 64, 94-100.

Shervani-Tabar M.T., Aghdam H.A., Khoo B.C., Farhangmehr V., Farzaneh B., 2013. Numerical analysis of a cavitation bubble in the vicinity of an elastic membrane. Fluid Dyn. Res. 45, 055503 (14pp). doi:10.1088/0169-5983/45/5/055503

Turangan C.K., Ong G.P., Klaseboer E., Khoo B.C., 2006. Experimental and numerical study of transient bubble-elastic membrane interaction. Journal of Applied Physics 100, 054910.

Vogel A., Lauterborn W., Timm R., 1989. Optical and acoustic investigations of the dynamics of laser-produced cavitation bubbles. J. Fluid Mech. 206, 299-338.

Vogel A., Schweiger P., Frieser A., Asiyo M.N., Birngruber R., 1990. Intraocular NdYAG laser surgery: light-tissue interaction damage range and reduction of collateral effects. IEEE J Quantum Electron 26, 2240-60.

Vogel A., Venugopalan V., 2011. Pulsed laser ablation of soft biological tissues. In: Optical thermal response of laser-irradiated tissue, second edition, ed. Welch A.J. and Van Gemert M.J.C., Springer pp. 551-615, DOI: 10.1007/978-90-481-8831-4_14 
Walsh, J.T., van Leeuwen, T.G., Jansen, E.D., Motamedi, M., Welch, A.J., 2011. Pulsed laser tissue interaction. In: Optical thermal response of laser-irradiated tissue, second edition, ed. Welch A.J. and Van Gemert M.J.C., Springer, pp. 617-649. DOI: 10.1007/978-90-481-8831-4_15

Zhang S., Duncan J.H., Chahine G.L., 1993. The final stage and collapse of a cavitation bubble near a rigid wall. J. Fluid. Mech. 257, 147-181. 